ÉGYPTE

monde arabe

\section{Égypte/Monde arabe}

$24 \mid 1995$

Anthropologies de l'Égypte 1

\title{
Du réalisateur au spectateur : la politique des feuilletons égyptiens
}

\section{Lila Abu-Lughod}

\section{(2) OpenEdition}

\section{Journals}

Édition électronique

URL : https://journals.openedition.org/ema/629

DOI : 10.4000/ema.629

ISSN : 2090-7273

\section{Éditeur}

CEDEJ - Centre d'études et de documentation économiques juridiques et sociales

\section{Édition imprimée}

Date de publication : 31 décembre 1995

Pagination : 43-58

ISSN : 1110-5097

\section{Référence électronique}

Lila Abu-Lughod, «Du réalisateur au spectateur : la politique des feuilletons égyptiens », Égypte/Monde arabe [En ligne], 24 | 1995, mis en ligne le 08 juillet 2008, consulté le 07 juillet 2022. URL : http:// journals.openedition.org/ema/629; DOI : https://doi.org/10.4000/ema.629

Ce document a été généré automatiquement le 7 juillet 2022.

Tous droits réservés 


\title{
Du réalisateur au spectateur : la politique des feuilletons égyptiens
}

\author{
Lila Abu-Lughod
}

\section{NOTE DE L'ÉDITEUR}

Traduit de l'anglais par Samia Rizq.

1 En 1980, circulait au Caire une plaisanterie typique du mépris que vouent les citadins au paysan de Haute-Égypte : un Sa îdi (Égyptien de Haute-Égypte) vient au Caire et veut acheter un téléviseur ${ }^{1}$. Il se rend dans un magasin d'électro-ménager et demande : «Combien vaut ce téléviseur dans la vitrine? » Furieux, le propriétaire lui crie : « Sors d'ici, stupide Sa'îdi!». II s'en va et, ayant revêtu la robe blanche et la coiffe des Saoudiens, retourne au magasin: «Combien vaut ce téléviseur dans la vitrine? " Le propriétaire lui crie : «Sors d'ici, stupide Sa'îdi!» II s'en va et, vêtu à l'européenne, retourne au magasin : «Combien vaut ce téléviseur dans la vitrine? " Même réaction : «Sors d'ici, stupide Sa î̀di ! » Intrigué, le pauvre homme demande alors : «Comment astu pu me reconnaitre? » Le propriétaire lui répond : «Dans la vitrine, ce n'est pas un téléviseur, c'est une machine à laver... »

Le manque de familiarité avec la télévision est ici interprété comme symptomatique de l'arriération des ruraux. La télévision est signe de modernité : produire des émissions requiert une technologie de pointe, les recevoir suppose de posséder un appareil coûteux. Bien que la télévision ait été introduite en Égypte en 1960, il a fallu compter avec la lenteur de l'électrification et attendre les salaires des travailleurs migrants, dans les années soixante-dix, pour que la plupart des ménages, en particulier hors des grandes villes, puissent disposer d'un téléviseur. On sait que l'industrie égyptienne du cinéma est l'une des trois plus importantes du monde, avec Bombay et Hollywood. L'Égypte est aussi le plus grand producteur de feuilletons et de séries télévisées arabes. Ses productions sont exportées vers la plupart des pays arabes et ses Studios privés, ses techniciens, ses acteurs et metteurs en scène comptent particulièrement sur l'énorme 
demande des pays du Golfe. Les programmes sont pratiquement tous produits au Caire. Or, la moitié des téléspectateurs potentiels, du moins en Égypte, vivent en zone rurale. L'origine urbaine des séries et feuilletons affecte immanquablement les types de programmes produits et la manière dont la population rurale y est représentée : le répertoire comporte souvent des personnages tels que le maire corrompu du village avec ses acolytes violents, reconnaissables à leur costume et leur dialecte inauthentiques ou au décor de leurs maisons ornées de tapisseries "primitives" représentant des scènes de village - tapisseries pour touristes et Égyptiens occidentalisés. La majorité des professionnels des médias sont issus de la classe moyenne et, quelle que soit l'idéologie politique qu'ils professent, leur origine exerce une réelle influence sur leur description des différentes classes sociales.

3 En tant qu'anthropologue, je me suis intéressée à la manière dont étaient représentés les ruraux et les travailleurs, ainsi qu'à la lecture que faisait cette population des séries et des feuilletons qu'elle regardait, mais à la production desquels elle n'avait pas participé.

4 Plus que tout autre média - et surtout là où il y a encore beaucoup d'illettrés -, la télévision apporte une variété d'expériences très denses de l'« ailleurs» (du non-local) dans un cadre des plus locaux, à savoir le foyer. Ainsi, lorsque Naguib Mahfouz, prix Nobel de littérature, déplore le déclin des cafés en expliquant que «les gens s'y rendaient pour écouler des conteurs qui jouaient d'un instrument de musique et racontaient l'histoire des héros populaires », et que "ces rencontres jouaient le rôle que jouent de nos jours les séries télévisées", il oublie que cette forme de divertissement suscitant un imaginaire de l'«ailleurs» (imaginary non-local worlds) n'était accessible qu'aux hommes ${ }^{2}$ et l'était davantage dans les villes que dans les villages. Or, avec la télévision, les femmes, les jeunes et les villageois ont accès aux histoires d'autres mondes au même titre que les hommes des villes.

5 En Égypte, un groupe actif de professionnels de l'industrie du spectacle considèrent que ces femmes, ces jeunes et ces villageois forment une classe de subalternes qu'il faut " éduquer ". S'appropriant le discours occidental sur le développement et le modulant à leur manière, ils se considèrent comme des guides devant conduire les masses vers la modernité et former, à travers leurs programmes, le citoyen modèle. Ils cherchent à « instruire» leur public, notamment par les émissions populaires. Leur foi dans l'impact de la télévision est stimulée par les-débats que ces séries suscitent chez les critiques et divers membres de l'intelligentsia urbaine.

6 Pourtant, si l'on regarde la place que la télévision occupe dans la vie de ce public, il semble bien qu'il subvertisse le projet des producteurs et leur échappe. Non que les téléspectateurs ruraux soient "traditionnels» ou ignorent ce qui est "moderne", comme le suggère la blague citée plus haut. Plutôt, leur manière de se situer par rapport à la modernité n'a rien à voir avec la vision que ces professionnels, citadins de la classe moyenne, tentent de promouvoir. Le message nationaliste est diffusé dans un espace social complexe où ce qui est typiquement local, aussi bien que le transnational, exerce une influence. D'une part, les gens vivent dans le monde local de leur expérience quotidienne, dont la télévision ne constitue qu'une petite partie. D'autre part, dans la mesure où les sociétés multinationales bombardent le marché égyptien de leurs produits, où les groupes islamistes affichent des identités et des loyautés dépassant le cadre national et où les élites regardent vers l'Occident, les nationalistes de la télévision ont fort à faire. 
La télévision dans le discours de la nation moderne

7 Dans le discours de certains piliers de l'industrie du spectacle en Égypte, la télévision a donc sa place dans une vision nationaliste et élitiste de la modernité. Lorsque Nûr alSharîf, acteur et metteur en scène, décrit les débuts de la télévision égyptienne, il souligne que, dans une ambiance générale marquée par l'idée de progrès national, le gouvernement avait pour projet d'utiliser les médias et les arts pour modifier l'opinion des masses sur la participation et la vie politiques ${ }^{3}$. Les films et les séries télévisées des années soixante s'inspiraient d'œuvres littéraires égyptiennes. Il s'agissait « d'éduquer le peuple, de l'éclairer et de l'impliquer dans la politique de la nouvelle révolution, en assurant le passage de la société égyptienne du féodalisme et du capitalisme au socialisme ». Il s'agissait encore, selon Nûr al-Sharîf, « d'aider la jeunesse égyptienne non instruite des régions rurales, de la province et des villes autres que le Caire et Alexandrie, nullement intéressée par la culture, à se familiariser avec les grands auteurs".

Ce souci déclaré d'« éduquer» les pauvres et tous ceux qui vivent hors des centres urbains trouve un écho chez Muhammad Fadil, un des premiers réalisateurs égyptiens, qui soutient que, dans les pays en développement touchés par l'illettrisme et l'inculture, la télévision ne doit pas être un simple moyen de divertissement; elle doit travailler à combattre cette inculture. Liant culture et responsabilité sociale, il définit la première en ces termes : " C'est être familiarisé avec les informations, apprécier l'art, la musique, le théâtre... La culture, c'est l'intérêt que porte l'individu aux problèmes d'autrui, elle provient du savoir. $»^{4}$ Définition qui n'est pas sans rappeler ceux de Lemer dans son portrait de «l'homme moderne ». Et Fadil de conclure que, puisque les séries et les feuilletons sont les émissions préférées du public, on doit y avoir recours pour l'instruire «à son insu »!

Un des aspects essentiels de cette construction du divertissement télévisuel comme un art sérieux, politiquement et socialement édifiant, est la comparaison avec les programmes commerciaux. Fadil distingue les séries et feuilletons que les spectateurs ont plaisir à regarder, de ceux qui produiront un effet bien longtemps après leur diffusion. Il critique ses collègues dont la production ressemble davantage à des " amuse-gueule» - pumpkin-seed serials, séries amusantes mais sans vraie valeur nutritive - qu'à un « repas consistant $»^{5}$. De son côté, Nûr al-Sharîf défend la politique télévisuelle mise en place par le gouvernement dans les années soixante, en soutenant qu'elle a permis de " faire naître un rêve national commun, non seulement en Égypte mais dans tout le monde arabe. Cela a rendu les gens plus enthousiastes et plus optimistes ». Il oppose cette époque à ce qui s'est produit par la suite, vers le milieu des années soixante-dix : les programmes ont commencé à être produits par des sociétés privées ayant le divertissement pour seul objectif, et liées par la nécessité de vendre leur production dans les pays conservateurs du Golfe, où la censure politique et morale est particulièrement restrictive ${ }^{6}$.

10 La forte audience de la télévision égyptienne au cours des cinq dernières années tient en grande partie au sérieux de certains feuilletons. Depuis 1988, lors du Ramadan - ce mois de jeûne musulman est une période de grande écoute -, les gens étaient cloués devant l'écran, fascinés par un feuilleton de Usama Anwâr 'Ukasha, quintessence du feuilleton qui n'est plus un «amuse-gueule ». Sous le titre Les Nuits de Hilmiyya, ce feuilleton met en scène des personnages de la banlieue traditionnelle cairote de Hilmiyya, à la fin des années quarante - l'Égypte était alors gouvernée par le roi Farûq 
et les Anglais -, et suit l'évolution de leurs fortunes et de leurs relations jusqu'à nos jours, insérant même, dans les derniers épisodes, la réaction égyptienne à la guerre du Golfe.

11 Bien que plusieurs séries et feuilletons égyptiens aient connu une large audience, suscité des débats et passionné les spectateurs, et que les productions locales soient généralement plus populaires que les productions importées, la diffusion de ce feuilleton exceptionnellement long et de grande qualité a été un événement culturel à l'échelle nationale ${ }^{7}$. Sa popularité ne s'est pas limitée aux milliers de téléspectateurs qui l'ont suivi tous les soirs, mais s'est étendue à l'intelligentsia, surtout intéressée par le contenu politique. Les mérites du feuilleton ont été débattus dans la presse et un intellectuel des plus en vue, Sayyid Yasin, l'a même utilisé comme métaphore des "capacités réelles de l'Égypte ». Dans un bref article publié par al-lqtisadi, grand hebdomadaire égyptien, Sayyid Yasin compare le succès du feuilleton, la qualité du texte, la compétence du metteur en scène et ses acteurs talentueux et consacrés, la mobilisation des auditeurs... aux échecs de l'activité politique actuelle. Il laisse entendre que l'Égypte a besoin d'un « texte » politique de meilleur niveau pour guider le «metteur en scène» (le chef de l'État), d'un plus grand respect des citoyens et de l'introduction de nouveaux « acteurs politiques $»^{8}$.

Le temps d'émission restant limité dans la journée, et la production télévisuelle, entièrement contrôlée par l'État, n'ayant été que très peu financée par la publicité jusqu'à une période récente ${ }^{9}$, il ne s'est pas développé en Égypte un équivalent des soap operas américains. En revanche, depuis la fin des années soixante, les feuilletons (musalsal) du soir, retransmis quotidiennement et comprenant entre quinze et trente épisodes, ont dominé les programmes. Ces feuilletons se distinguent des soap operas, diffusés dans la journée ou aux heures de grande écoute, par le fait que leur diffusion s'étale sur une durée limitée et que le dénouement de l'intrigue est reporté au dernier épisode ${ }^{10}$.

Le feuilleton dont nous parlons est un produit hybride. Étalé sur une durée de cinq ans et comprenant beaucoup plus d'épisodes que les feuilletons antérieurs, son dénouement a été constamment repoussé. Aussi Les Nuits de Hilmiyya ont-elles favorisé le type d'attachement aux personnages que créent les soap operas. En suivant les mésaventures et les succès de certains d'entre eux de leur enfance à l'âge adulte, et la vie de certains autres à travers des événements marquants (mariages, divorces, décès, emprisonnements, carrières) et jusqu'à un âge avancé, les téléspectateurs sont invités à jouir du plaisir qu'offre ce genre de production : saisir le sens d'une scène parce qu'ils connaissent l'histoire des personnages ${ }^{11}$. Une sorte de complicité $s^{\prime} e s t$ donc créée avec le temps, les téléspectateurs étant profondément familiarisés avec l'histoire personnelle des héros et les rapports complexes qui les lient. D'autant que les anciens épisodes sont rediffusés avant la présentation des nouveaux et qu'en 1993, l'ensemble du feuilleton, maintenant achevé, a été retransmis tous les dimanches soir ${ }^{12}$.

Le feuilleton Les Nuits de Hilmiyya est cependant radicalement différent de tous les soap operas américains du fait qu'il se situe dans un contexte historique donné. Il décrit la vie personnelle des individus mais, plutôt que de faire simplement progresser le récit au fil des événements qui surviennent dans leurs relations, il traite de thèmes moraux (loyauté, trahison, corruption, désirs contrariés, erreurs tragiques...) insérés dans un contexte qui lie la vie des individus aux événements politiques nationaux. Ainsi fait-il 
ce qu'aucune série américaine ne fait habituellement : fournir un commentaire social et politique de la vie égyptienne contemporaine ${ }^{13}$.

Surtout, notre feuilleton met en avant le thème de l'unité nationale. À l'exception de rares individus foncièrement égoïstes et corrompus, les personnages de différentes classes et opinions politiques sont présentés comme fondamentalement bons et patriotes. Nous prenons en pitié ceux qui s'égarent à la suite de revers amoureux ou politiques. Ils finissent cependant par prendre conscience de leurs erreurs : leur amour pour l'Égypte les empêche de poursuivre dans la mauvaise voie, celle du matérialisme, de l'immoralité ou de la corruption. Même le jeune extrémiste religieux (le premier à être apparu dans un feuilleton télévisé égyptien) est une figure sympathique, dépeinte comme appartenant à une génération que l'absence d'esprit national a dévoyée ${ }^{14}$.

Des individus «non éclairés »?

Pour étayer l'idée que la télévision dépasse le simple divertissement, les professionnels de la culture intéressés par les questions sociales et jouissant d'une "conscience politique» ont décidé que leur public serait celui de gens ayant "besoin d'être éclairés ». Mais comment savoir si les feuilletons qui lui sont destinés touchent ce public? Je me fonderai sur un travail ethnographique que j'ai mené en Égypte dans les années quatre-vingt-dix pour montrer comment deux, au moins, des groupes visés (femmes de la classe ouvrière et villageois) semblent échapper au «filet » de leurs bonnes intentions.

U. A. 'Ukasha pourrait éprouver quelque soulagement en découvrant que bon nombre de ces "analphabètes" sont à l'abri des «amuse-gueule " que sont certaines séries américaines comme The Bold and the Beautiful. Ce soap opera destiné en principe à un public américain "subalterne", les femmes au foyer - mais suivi par nombre d'étudiants - a captivé, en Égypte, aussi bien les hommes que les femmes appartenant à la classe moyenne, voire à l'élite intellectuelle: il fallait en effet, pour apprécier, connaître l'anglais ou pouvoir lire les sous-titres en arabe.

De plus, il est rassurant pour l'auteur de savoir que maints des feuilletons produits par des collègues moins concernés qu'il ne l'est lui-même par l'aspect social sont de qualité tellement médiocre que personne ne s'y intéresse. Sans doute serait-il moins satisfaisant pour lui de découvrir que certains spectateurs résistent à ses messages sociaux édifiants, ce qui ne les empêche pas de considérer Les Nuits de Hilmiyya comme le meilleur feuilleton jamais produit à ce jour. Prenons un exemple: le feuilleton présente plusieurs figures féminines confrontées au dilemme commun à plusieurs générations de femmes. Les plus âgées endurent de mauvais traitements de la part de leurs époux - mariage clandestin avec une autre, déceptions diverses... Les plus jeunes se débattent entre carrière et mariage. Le feuilleton glorifie l'instruction : la plupart des filles de la classe ouvrière y poursuivent des études universitaires, la fille d'un propriétaire de café et d'une danseuse devient professeur d'Université, celle d'un ouvrier est médecin. En général, les femmes sont indépendantes, capables de prendre des décisions. Le "féminisme d'État» de l'époque nassérienne y est glorifié : l'épouse obstinée d'un ouvrier conseille à une amie de défendre les droits que leur a donné « Gamal » ${ }^{15}$.

Cependant, interrogées sur ce qu'elles ont particulièrement apprécié dans le feuilleton, plusieurs femmes de milieu populaire, employées de maison au Caire, répondent spontanément que c'est, non pas le contenu politique et social, mais le personnage de Nazik Hanem, cette aristocrate duplice, femme fatale magnifiquement vêtue et qui joue 
le principal rôle féminin. Une jeune femme abandonnée par son mari et élevant seule ses deux enfants déclare que si tout le monde regarde Les Nuits de Hilmiyya, c'est " pour Nazik : elle est dure ; elle a eu quatre maris, elle ne se laisserait pas guider par qui que ce soit».

Une femme plus âgée voit dans Nazik Hanem "une femme capricieuse qui ne se satisfait pas d'un seul type ; elle s'est mariée plusieurs fois, elle est têtue et obstinée ; à cause de ses désirs, elle a perdu sa fortune. (...) Et Hamdiyya, la danseuse, vous l'avez vue?» Elle rit en imitant un geste arrogant typique de cette danseuse orientale devenue propriétaire de cabaret.

21 Tels sont les deux types de femmes séduisantes, ayant peu de sympathie pour les nationalistes, qui ont réussi à « embobiner les hommes » et qui ne se comportent pas en «femmes respectables» malgré les adjurations de leurs enfants, de leurs ex-époux et d'autres membres de la famille. Ce sont également ces deux catégories de personnages qui ont subi les pires déchéances. Trompée par un homme qu'elle espérait épouser, la danseuse s'adonne à l'héroïne. Elle a tout perdu et ne sait plus où aller. La fin de Nazik Hanem, brillamment interprétée, est plus complexe. Ne pouvant admettre les effets de l'âge - même après avoir été ruinée par son quatrième époux, un homme, plus jeune qu'elle et qui l'a rejetée -, elle s'habille comme une jeune fille, dissimule ses cheveux gris sous une perruque, flirte avec un étudiant de vingt ans. Désapprouvée par les siens, elle se réfugie dans la dépression.

22 Aucune des femmes ayant exprimé leur admiration pour Nazik ou la danseuse n'ont fait allusion à la dimension morale de telles fins. Elles semblaient plutôt trouver un plaisir indirect à déceler chez ces femmes un défi au système moral qui laisse en paix les femmes "convenables». En fait, ces femmes qui travaillent comme domestiques sentent leur respectabilité menacée par la nécessité de travailler hors du foyer, surtout en exerçant un tel métier. Elles se battent quotidiennement pour être respectées, cachent à leurs voisins, parfois même à leurs proches, le type de travail qu'elles exercent et ont toutes adopté le hijâb (voile de tête) qui en Égypte, est devenu signe de piété et de respectabilité dans la classe moyenne.

U. A. 'Ukasha pourrait se reconnaître quelque responsabilité dans cette résistance au féminisme et à la morale nationalistes. Pour plaire au grand public et dépeindre des personnages vertueux, modernes et patriotes, les talentueux producteurs de feuilletons en arrivent souvent à tomber dans une imagerie populaire analogue à celle des soap operas américains, ou à avilir " l'autre » - généralement parodié - pour mieux faire ressortir le contraste. Ce qui peut nuire à des personnages aussi excessifs que Nazik Hanem ou que le scélérat ignorant et corrompu d'un autre succès de l'auteur, Al-Raya al-Bayda' (Le drapeau blanc), qui a pourtant ravi la vedette aux protagonistes " sérieux " et... assommants ${ }^{16}$.

Seule une femme d'un certain âge a commenté, plus que ne l'avaient fait les critiques eux-mêmes, l'idéologie nationaliste explicite du feuilleton. Selon elle, Les Nuits de Hilmiyya sont "contre Sadate", c'est pourquoi l'on y montre les conséquences de l'ouverture économique (par exemple, importation de viande en conserve qui se révèle être de la nourriture pour chats). Elle affirme clairement ne pas partager la position de 'Ukasha et ajoute: « De nos jours, tout le monde cherche à s'enrichir. Il y a beaucoup d'argent. Pour nous tous.» Les opinions politiques les plus diverses circulent en Égypte... et rien ne permet de présumer que des spectateurs " peu éclairés » attendent passivement les leçons que les feuilletons sont censés leur apprendre. 

feuilletons télévisés. Ils auraient pu manifester un désaccord politique, s'émerveiller de voir des personnages intransigeants vivre comme ils ne peuvent le faire eux-mêmes. Or, ils n'ont accepté le point de vue moral qui leur était présenté que lorsque celui-ci trouvait une résonance dans leur propre univers, Cela se manifeste clairement dans la réaction des femmes de milieu modeste, où règne généralement un certain conservatisme moral concernant la famille et le rôle de la mère tel qu'il est habituellement présenté dans les feuilletons égyptiens, et dans Les Nuits de Hilmiyya en particulier. Conservatisme qu'illustrent bien les propos de cet admirateur de Nazik Hanem tentant d'expliquer pourquoi Zohra (la fille de Nazik) n'a jamais trouvé le bonheur: "La pauvre! C'est parce que sa mère ne s'en est pas occupée. Elle l'a abandonnée à son père lorsqu'elle était encore bébé. Quand la petite tombait malade et que la nouvelle épouse la ramenait chez Nazik en lui disant : "Prends ta fille et serre-la fort dans les bras", Nazik refusait. Zohra n'a jamais connu l'amour maternel. Elle devait compter sur elle-même. Voilà pourquoi, plus tard, elle s'est laissée tromper... »

Les spectateurs de Haute-Égypte interrogés pour les besoins de cette étude ne montrent pas davantage de souplesse que les femmes de la classe ouvrière urbaine quant au contenu moralisateur des feuilletons. Non que la télévision leur soit peu familière : dans le village proche de Louxor où j'ai mené mon enquête, chaque ménage possédait un téléviseur, souvent noir et blanc et ne permettant qu'une mauvaise réception. Les familles les plus riches avaient un grand téléviseur couleur. En tête de la liste des vœux de chaque famille pauvre venait le souhait de vendre un bout de terrain ou d'économiser pour en avoir un également. Rares étaient les soirées où la télévision restait silencieuse : un décès dans le quartier ou dans la famille, un malade dans la maison, s'il avait des visiteurs, ou encore, cause la plus fréquente et la plus commune, une coupure d'électricité.

Plus précisément, l'impact de feuilletons comme Les Nuits de Hilmiyya est infléchi par la manière dont les villageois consomment les programmes. Comme leurs homologues urbains, ceux que j'ai rencontrés étaient capables de faire une lecture sélective des feuilletons, sélection souvent nécessaire en raison de l'écart important opposant les « réalités » mises en scène dans le feuilleton et la vie que l'on menait dans le village : d'un côté, les élégantes vedettes blondes de The Bold and the Beautiful dans leurs somptueux bureaux et leurs maisons luxueuses; de l'autre, de laborieux travailleurs vivant dans des maisons en briques d'argile, aux portes assez larges pour laisser entrer le bétail dans les enclos. Or, les personnages d'un feuilleton comme Les Nuits de Hilmiyya leur sont a peine plus proches. Les feuilletons de ce type se déroulent presque tous en milieu urbain et traitent exclusivement de problèmes urbains, voire des seuls problèmes des classes favorisées ${ }^{17}$.

La manière dont une famille de condition modeste regarde la télévision montre bien l'abîme qui sépare sa vie de celle que l'on voit sur le petit écran, ainsi que l'interprétation sélective opérée par les femmes en regardant les programmes. Un soir, alors que Yamna, mère de famille pleine d'entrain mais exténuée, prépare le repas du soir, aidée par sa sœur, commence le feuilleton Hobb fi haqiba diplomasiyya (L'amour dans une valise diplomatique). Toute la journée, la sœur a aidé cette mère surmenée qui doit cuire le pain et surveiller les enfants tout en allant chercher le fourrage pour les bêtes. Entre la fièvre de l'aîné, la rougeole qui a frappé les quatre filles, les examens de fin d'année des trois garçons, les dépenses et la fatigue occasionnées par une récente 
visite à l'hôpital d'Assiout dans l'espoir de trouver un remède à la toux asthmatique du père, grand fumeur, et la hausse du prix de la farine annoncée la veille par le gouvernement, tous se demandent comment ils vont pouvoir s'en tirer. Le feuilleton qu'ils regardent présente une riche famille de diplomates et, entre autres personnages, des professeurs de ballet, des femmes médecins, des journalistes et personnalités de la radio confrontés à des problèmes de carrière.

Pendant que Yamna cuisine, sa sœur lui résume les événements du feuilleton en mettant l'accent sur la dynamique de la famille, comme les villageois ont l'habitude de le faire quand ils racontent leur propre vie: divorces, disputes, abandons, mariages ratés. Elle ne manque pas de relever le contenu moral du feuilleton concernant la femme et la famille: importance du rôle de la mère dans l'éducation des enfants, conséquences fâcheuses pour eux si leur mère les délaisse ou s'intéresse davantage à elle-même, à son mariage ou à sa carrière.

Plusieurs des "problèmes féminins » évoqués dans ce feuilleton, écrit et mis en scène par une des rares femmes metteur en scène en Égypte, sont traités sous un angle psycho-social étranger à ces femmes: problèmes d'ordre psychologique tels qu'une paralysie psychosomatique, que l'amour peut guérir, hommes incapables de s'engager dans le mariage de crainte de perdre leur liberté, mères qui pleurent parce que leurs enfants ne s'ouvrent pas à elles, psychiatres soignant des toxicomanes de milieux aisés et instruits. Ainsi, dans leurs discussions, les femmes ignorent complètement les aspects du feuilleton qui ne font pas parte de leur expérience.

31 L'étude ethnographique menée dans ce village m'amène à penser que ses habitants constituent un public cible fermé aux messages modernisateurs de l'élite culturelle, ceci en raison de la facilité avec laquelle la télévision a été intégrée à leur vie quotidienne. Bien qu'il soit difficile de dire clairement comment cela s'est produit, je soutiens que la télévision a créé son propre monde, un monde qui ne représentait pour les villageois qu'une partie - passionnante il est vrai - de la vie quotidienne. L'expérience vécue à travers la télévision est venue s'ajouter à ce qui existait déjà mais n'a pas pu le supplanter. Le monde de la télévision est perçu non comme une évasion dans l'imaginaire mais comme une sphère en soi, ayant ses créneaux habituels et supposant une attitude particulière. C'est un monde de connaissances qui donne lieu à un échange d'informations: les adolescents ont une connaissance encyclopédique des films et des feuilletons égyptiens et les spectateurs en savent long sur la vie privée et sur les rôles précédemment interprétés par les acteurs et actrices du feuilleton.

L'attitude des villageois envers les vedettes répond à une question plus vaste: quelle influence les feuilletons exercent-ils sur le public? En pariant de ces vedettes, les villageois disent «nos » vedettes comme si, en quelque sorte, elles leur appartenaient en tant que spectateurs, mais n'utilisent pas le «nous » qui les assimilerait à eux. De même, les spectateurs peuvent considérer comme un dû la prestation des stars tout en gardant leurs distances par rapport au feuilleton: les stars sont là pour "notre " plaisir, mais le feuilleton décrit la vie des autres, ceux qui ont des problèmes différents, respectent des règles différentes et n'appartiennent pas à la "communauté morale " locale. Ce que font ces autres a donc peu d'effet sur ce que nous faisons ou sur notre manière de vivre. Essayer d'arrondir le salaire mensuel du père (130 livres égyptiennes) en plantant du trèfle pour le bétail, se débattre pour élever les enfants, assurer leur scolarisation et leur mariage, revendiquer ses droits de succession ou tirer de l'argent de la famille, préserver sa réputation et faire face à la belle-famille et aux voisins 
jaloux, telles sont les préoccupations quotidiennes des villageois. S'ajoute à cela le plaisir que procurent les visites chez les amis ou la famille, les obligations sociales dont on s'acquitte généreusement, la réussite aux examens, la vente d'une bête ou encore le fait d'avoir une bonne récolte ou de porter des vêtements neufs aux fêtes religieuses.

Même lorsqu'un feuilleton tente de reproduire la vie au village, comme dans Hasad alHobb (Les fruits de l'amour), écrit par une femme progressiste politiquement engagée, le public rejette les problèmes qui y sont soulevés comme n'étant pas les siens. Il prend plaisir à regarder le feuilleton, se retrouve dans le dialecte et parfois dans les vêtements, mais prétend ne pas être concerné par le thème principal - la vendetta qui se passe toujours ailleurs que chez soi, quelque part en Haute-Égypte. Cela se produit " peut-être à Sohag ", proposent certains villageois, alors qu'on m'aura fait par ailleurs le récit de vendettas qui ont eu lieu dans leur village il y a à peine quelques décennies.

De même, lorsqu'il s'agit de classer, ces « autres » mis en scène à la télévision, l'altitude des spectateurs diffère totalement de celle qu'ils adoptent envers leurs voisins ou les membres de leur famille. Alors que dans ce cas ils exercent leur esprit critique et portent des jugements, ils ont sur les « autres » ce regard à la fois curieux et neutre que portent en général les villageois sur les touristes, les archéologues et les chercheurs étrangers qu'ils côtoient depuis le début du siècle.

Je ne soutiens pas ici que les villageois entendent compartimenter la « modernité » que présentent les feuilletons de manière à préserver une communauté traditionnelle et statique qui n'aurait pas, en quelque sorte, été touchée par cette "modernité ». Au contraire, tandis que le village peut apparaître, à un regard extérieur, comme une réalité " pittoresque ", avec ses maisons en terre, ses palmiers se balançant au vent, ses ânes, ses chameaux chargés de trèfle ou de canne à sucre, ses hommes qui travaillent aux champs ou vont nu-pieds dans les canaux d'irrigation boueux, ses femmes dans leurs longues robes noires, balançant leurs baluchons sur la tête..., il n'y a aucun aspect de la vie quotidienne qui n'ait été façonné par la «modernité ». Contrairement à la blague qu'on raconte au Caire, tout le monde arrive à s'arranger pour avoir non seulement la télévision, mais aussi la machine à laver, le réfrigérateur, le ventilateur et la pompe à eau. Au village, la télévision fait partie d'un mélange complexe et les expériences et les images qu'elle présente sont intégrés avec une aisance surprenante comme des éléments discrets - mais non écrasants - de ce mélange.

36 Je ne veux pas dire par là que, d'une manière générale, la télévision n'a pas transformé la vie sociale et l'imaginaire. Il existe au moins trois domaines dans lesquels des recherches ethnographiques attentives révéleraient d'importantes transformations.

37 En premier lieu dans la vie sociale: les visites sont moins fréquentes depuis que les familles passent la soirée devant le petit écran. Qui plus est, la télévision a pu contribuer à augmenter le nombre d'« expériences " partagées par des générations différentes et par des personnes de sexe différent.

En second lieu, la télévision a changé la nature même de l'expérience «dramatique »: des professionnels égyptiens, pour expliquer le plaisir des téléspectateurs, mettent en valeur l'élément de continuité : les feuilletons auraient le même rôle que les « histoires racontées par la grand-mère à ses petits enfants pour les endormir ", ou que les Mille et une Nuits, où le narrateur s'arrête au moment le plus palpitant pour tenir son public en haleine jusqu'au lendemain "; ils évoquent encore la tradition nord-africaine où «le poète musicien, le griot, chante ses poèmes au son de la rabâba en s'arrêtant chaque 
jour à un point palpitant du récit de 'Antar ou d'Abu-Zayd al-Hilâli » ${ }^{18}$. Ces analogies méconnaissent une caractéristique des feuilletons télévisés notée par Raymond Williams (1989) concernant leur rôle dans la théâtralisation de l'expérience du monde elle-même.

Troisième domaine où la télévision contribue sans doute à transformer l'expérience : celui de l'identité, en facilitant de nouvelles affiliations et de nouvelles identifications. Les villageois n'ont-ils pas le sentiment d'appartenir à la même «communauté imaginée $»^{19}$ de citoyens ou de consommateurs, sachant qu'ils sont en train de suivre le même programme que tout le monde, et à la même heure, à travers le pays ?

Toutefois, il ne s'agit pas ici d'identifier ou d'étudier les effets généraux de la télévision sur l'expérience des téléspectateurs, mais plutôt de cerner les effets de certains types d'émissions à succès sur le public qu'elles visent. Deux facteurs contribuent à atténuer l'impact de feuilletons comme Les Nuits de Hilmiyya. D'une part, ce feuilleton n'est qu'un élément parmi d'autres dans un flot d'émissions ${ }^{20}$ comprenant des films, des messages publicitaires, des émissions religieuses, des émissions sportives, des programmes pour enfants, des informations, etc. D'autre part, les messages transmis se trouvent très souvent pondérés, voire contredits, par la réalité quotidienne rigoureuse à laquelle sont confrontés aussi bien les villageois que les ouvrières du Caire. Ces réalités sont tout à la fois résolument locales et transnationales.

Alors que Les Nuits de Hilmiyya et les feuilletons du même genre condamnent le consumérisme et le matérialisme encouragés, dans les années soixante-dix, par la libéralisation économique et le rejet du socialisme, les publicités qui précèdent le feuilleton et le mode de vie des classes moyenne et riche tel qu'il y est représenté véhiculent le message inverse. Chez les enfants d'âge scolaire, les biens disponibles dans le village, proche de Louxor, où j'ai travaillé, sont associés aux messages télévisés et inspirent le désir d'acheter une marque particulière de confiserie ou de chaussures de jogging. Pour les citadines des classes populaires, la disponibilité de tels produits est bien sûr plus forte encore.

Par ailleurs, U. A. 'Ukasha et les autres professionnels « éclairés » écartent de l'intrigue toute description positive des différents aspects sous lesquels l'alternative islamique est proposée au peuple égyptien. Pourtant, la promotion de l'identité et du savoir islamiques à travers les émissions religieuses entre en interaction avec l'expérience que vivent les adultes dans les mosquées neuves ou rénovées qui ont bénéficié d'un soutien enthousiaste, et avec celle que connaissent les enfants dans les écoles, institutions étatiques laïques qui sont, ironie du sort, le lieu privilégié d'exercice de la pression religieuse, surtout en milieu rural. Dans le village où a été menée cette étude, le port du voile est obligatoire et les enfants contraints de suivre les cours d'enseignants scrupuleusement religieux.

De plus, pour les villageois de Haute-Égypte comme pour les femmes de condition modeste vivant au Caire, il n'est guère d'éléments qui viennent confirmer les messages édifiants sur la culture, la responsabilité sociale et l'unité nationale. Avec sa vision séculière d'une Égypte moderne, pleine de citoyens vertueux, patriotes, unis au-delà des classes par leur amour de la patrie, Les Nuits de Hilmiyya tente d'intégrer les classes défavorisées dans son monde « moderne».

44 Or, ces dernières sont déjà intégrées dans la modernité égyptienne, mais de manière différente. Les enfants qui chantent chaque matin l'hymne national sont en quelque sorte ouverts aux messages des feuilletons télévisés. Certains rêvent d'un avenir 
irréalisable, comme cette jeune villageoise sur le point de terminer ses études à l'école d'agriculture, et qui attend le jour où le gouvernement lui fera don d'un terrain dans le Sinaï, qu'elle pourra cultiver. D'autres, plus âgés, estiment que leur région, la HauteÉgypte, a longtemps été marginalisée et exploitée par le Nord.

Pour eux, la "nation », ce n'est pas ce qu'en disent les chants patriotiques, mais ce qui transparaît dans les pratiques de la bureaucratie, d'une police ou d'une armée qui les a le plus souvent maltraités. L'« union du riche et du pauvre », idéalisée dans Les Nuits... se trouve annihilée par la réalité : les classes défavorisées savent comment les plus fortunés parviennent à "acheter" divers privilèges ou à contourner les règlements. Pour les citadines de condition modeste, avoir à résoudre le double problème qui consiste à travailler tout en conservant sa dignité par le port du voile ne confirme nullement l'image de la nation telle qu'elle leur est présentée.

Le problème réside enfin dans le fait que le genre de "modernité » présentée par les feuilletons comme une « vision de l'Égypte » dépend du statut social du spectateur et de la possibilité qui lui est offerte d'accéder à un certain type d'enseignement et à certaines carrières. La "modernité " à laquelle participe le public "analphabète " auquel s'adressent ces messages, c'est la forrne la plus commune du monde postcolonial : la modernité de la pauvreté, les désirs de consommation, le sous-emploi, la mauvaise santé et le nationalisme religieux.

\section{NOTES}

1. Version abrégée et modifiée d'un article intitulé « Objects of Soap Opera », à paraître dans Worlds Apart: Modernity through the Prism of the Local, ed. Daniel Miller, Routledge, Londres, 1995. Je remercie l'American Research Center in Egypt, l'ACLS/SSRC Joint Committee on the Near and Middie East et la Presidential Fellowship de l'université de New York pour l'aide financière qu'ils m'ont accordée pour mon travail de terrain en Égypte en 1989-90 et 1993. Je tiens également à remercier Maha Mahfouz 'Abd alRahman et Hala Abu Khatwa pour leur collaboration. Les opinions émises ici n'engagent toutefois que ma seule responsabilité.

2. Souligné dans Hedges C., "In Cairo Now a Coffee Shop is Just a Shop », New York Times, 03/08/92.

3. Entretien avec l'auteur, $22 / 07 / 90$.

4. Idem. $15 / 04 / 90$.

5. Idem.

6. Idem, $22 / 07 / 90$.

7. Il arrive souvent que les productions nationales touchent un public plus vaste que celui des productions importées. Pour un exemple européen, voir Silj A., East of Dallas: The European Challenge to American Television, British Film Institute, Londres, 1988.

8. Yasin S. «Cultural Papers: Hilmiyya Nights' and Political Activily », al-lqtisadi, 09/07/90, p. 96-97. 
9. Avec l'augmentation des produits disponibles sur le marché, on assiste à une forte compétition et, dans un pays où l'illettrisme est élevé, la publicité télévisée est efficace. D'après Aïda Nasr (Cairo Today, novembre 1992, p. 98), l'industrie de la publicité télévisée a rapporté 50 millions de livres égyptiennes, montant de la commission qui est de 15 à $20 \%$.

10. Cf. Geraghty C., Women and Soap Opera, Cambridge, Polity Press, 1991.

11. Ibid., p.15.

12. Selon 'Abd al-Nur Khalîl, « Le Ramadan de la télévision a captivé le public » (alMusawwar $n^{\circ} 3518,13 / 03 / 92$, p. 48-49), et les acteurs ont si bien incarné leur personnage qu'à la fin du feuilleton, plusieurs se sentaient prisonniers de ce dernier. Comme dans les soap operas américains, certains acteurs se sont désistés avant la fin du feuilleton ; il a donc fallu les remplacer et le public devait s'y adapter, dissocier le personnage de l'acteur qui l'interprétait.

13. La seule exception, diffusée récemment par la télévision américaine, c'est Murphy Brown : la campagne de la famille Bush en faveur des valeurs morales est attaquée en représailles contre l'intervention du vice-président Dan Quayle. qui condamne la décision de l'un des personnages du feuilleton de faire un enfant illégitime. Le fait que cette affaire ait fait tant de bruit dans la presse et ait été l'objet d'un documentaire à la $\mathrm{BBC}$ montre à quel point il est peu habituel que les questions politiques soient explicitement traitées dans les programmes de la télévision américaine.

14. Sur l'exclusion de la perspective du mouvement islamique des feuilletons télévisés, voir Abu-Lughod L, « Finding a Place for Islam: Egyptian Television Serials and the National Interest », Public Culture, vol. 5, t. 3, p. 493-513, 1993.

15. Sur le féminisme d'État et sa fin en Égypte, voir Hatem M., « Economic and Political Liberalization in Egypt and the Demise of State Feminism », International Journal of Middle East Studies $\mathrm{n}^{0} 24$, p. 231-251.

16. Pour plus de détails sur ce feuilleton, voir Abu-Lughod L., « Finding a place... », op. cit.

17. Selon l'étude de Soha 'Abd al-Qadir (« The Image of Women... », op. cit., p. 36), les réalisateurs de feuilletons ont un parti pris en faveur des classes urbaines privilégiées. Sur un échantillon de 12 pièces courtes et de 14 feuilletons diffusés sur une durée de 6 mois en 1980, aucun ne se déroulait en milieu rural.

18. Propos tenus par Nûr al-Sharîf (entretien réalisé par l'auteur le 22/07/90).

19. Formulation qui fait référence à l'ouvrage de B. Anderson, Imagined communities, Verso, Londres, 1983.

20. Williams R., " Drama in a Dramatised Society », Raymond Williams on Television, A. O'Connor ed., Routledge, Londres, 1989, p. 3-13.

INDEX

Mots-clés : télévision, feuilleton, anthropologie 
AUTEUR

LILA ABU-LUGHOD

Université de New York 